\title{
Torsion of the Pedicle in Hydrosalpinx, and other morbid conditions of the Fallopian Tube.*
}

By R. Hamilton Bell, M.A., M.B., B.C. (Cantab.), M.R.C.P. (Lond.), Physician to Out-patients, Samaritan Free Hospital; Obstetric Tutor and Registrar, St. Thomas's Hospital.

Torsion of the pedicle in tumours of the Fallopian tube is a rare phenomenon. To Mr. Bland-Sutton we owe the first recorded observation. It was a hydrosalpinx with its pedicle twisted three and a half times, and was removed by Mr. Henry Morris, in 1891. The specimen is preserved in the Museum of the Royal College of Surgeons. Since that date very few cases have been observed in England, or at any rate published, and the subject has never aroused much interest or attention. The case is very different on the Continent. In France observations of the phenomenon are constantly being made, and at the Société d'Obstétrique de Paris are the subject of frequent discussion. In addition, Hartmann and Reymond, Maillard, and Cathelin have written articles, with a record of all previously observed cases, and a full discussion of the clinical and pathological phenomena.

In Germany also, numerous cases have been recorded, and Praeger, in the Archiv für Gynäkol., 1899, collected twenty cases from the literature, and added two of his own.

My attention was directed to the subject by the occurrence of a case under the care of Dr. Cullingworth in St. Thomas's Hospital. It was the first case of the kind which had come under his personal observation, and he suggested to me that it would be valuable to record it, and at the same time to look up the records of other published cases.

On searching the literature $I$ soon found that the cases, though rare, were by no means so unique as was thought only a few years ago. Hartmann and Reymond, in 1898, collected eleven cases, Praeger in the following year reached twenty-two, and finally Cathelin, at the end of his article "De la Torsion des HydroSalpinx," published in 1901, added a table of forty-one observations. A few of these might perhaps be disputed. He includes, for example, a specimen of axial rotation of a right-sided parovarial cyst with attached right ovary and Fallopian tube distended by hæmorrhage. This case was published by Napier in the Transactions of the Obstetrical Society, 1892, vol. xxxiv. Clearly, though closely akin

* Read at a Meeting of the Obstetrical Society of London, April 6th, 1904. 
to the cases we are considering, it is only in a very loose classification included amongst them.

Hartmann and Reymond show the same laxity as Cathelin in this respect, and in their article headed "La Torsion des Salpingites," they include a case of twisting of a healthy tube, and another where the main cystic mass contained 500 grammes of blood, and was very probably ovarian rather than tubal.

It has fallen to the lot of most men who have operated on a considerable number of ovarian tumours to come across an ovarian cyst with twisted pedicle associated with a hæmatosalpinx, produced by the torsion of a hydrosalpinx which was adherent to the ovarian cyst. One such case occurred in St. Thomas's Hospital in June, 1898. But here the main mass is ovarian, and the associated hæmatosalpinx is a mere accessory. Such cases should therefore not be classified with those we are considering here, in which the torsion occurs in connection with primary tumours of the Fallopian tube, and in which the ovary may, or may not, be involved in the twist. Praeger excludes all such cases from his list, and he also particularly excludes one published by Montgomery (American Journal of Obstetrics and Gynocology, vol. ix.) of a cyst in the broad ligament closely connected with the tube, a case similar to Napier's mentioned above, and included by Cathelin.

But even if we are careful to exclude these cases the number of recorded observations has now reached fifty at the least. I had intended to draw up a table of all the cases, but abandoned this on discovering that it had already been done by Cathelin as recently as 1901. I will, however, before giving details of the case on which this paper is based, mention shortly those which I have discovered in the literature, recorded since 1901, or which had escaped Cathelin's notice. These are twelve in number.

1. Praeger. Archiv für Gynäkol., 1899, p. 583.

A married woman, aged 22, one child. Pelvic tumour, placed on the right side, and noticed nine months before operation. Severe abdominal pain, vomiting and constipation.

Diagnosis. Tubal or ovarian tumour, either intra-ligamentary or adherent.

At operation tumour found arising not from right but left side. Tubal in origin, dark-red in colour, and adherent. Pedicle twisted twice in the direction of the hands of a watch. Pedicle $7 \frac{1}{2} \mathrm{~cm}$. long; left ovary drawn into the twist. Below the tumour was a hrmatocele the size of a fist. Recovery.

2. Praeger. Archiv für Gynäkol., 1899, p. 584.

A married woman, aged 35, one child. Tumour on left side of abdomen, 
first discovered one year before operation; later 12 weeks' amenorrhca, with sickness, severe abdominal pains, retention of urine, constipation, etc. Tumour in left iliac fossa, tender, fluctuating. Below this four months' pregnant uterus.

Diagnosis. Left-sided ovarian cyst with twisted pedicle, associated with four months' pregnancy. At operation, dark-red cystic swelling, which turned out to be a left tube, with a pedicle twisted twice in the direction of the hands of a watch. Ovary not involved. Contents, fluid blood and detritus. Tube between twist and uterus very thin. Recovery. Pregnancy not interrupted.

3. Pozzi. Comptes Rendus de la Soc. d'Obstét. de Gynécol. et de Padiat. de Paris, April, 1900.

A right tubal pregnancy, three and a quarter months, with torsion of the pedicle; left hydrosalpinx. Married woman, aged 33, one previous pregnancy, seven years ago; last period Jan. 4th, 1900. On Jan. 19th, severe pains in lower abdomen, and slight hæmorrhage. This hæmorrhage continued daily till the operation, April 2nd. On Feb. 23rd another severe attack of abdominal pain, with vomiting. Tenderness of the lower abdomen. Per vaginam a cystic tumour was felt on the left side, but at the operation this was found to be attached to the right cornu of the uterus by a pedicle which had one complete turn in the inverse direction of the hands of a watch. The twist was not very tight, so that the circulation had not been interrupted in the tube, and the orum had continued to grow. Ovary not adherent to the tube, but involved in the torsion.

(This case is mentioned by Cathelin, but not included in his table, because it is a tubal pregnancy and not a hydrosalpinx. He however includes one clear case of tubal pregnancy.-A Martin, Centralbl. für Gynäkol, 1893).

4. Pinard and Paquy. Comptes Rendus de la Soc. d'Obstét. de Gynécol. et de Padiat. de Paris, October, 1901.

Torsion of the pedicle of a right hydrosalpinx, coinciding with a four months' pregnancy. Married woman, aged 26, one previous pregnancy. Several severe attacks of abdominal pain in the course of the present gestation. Vomiting in the last attack. Tender cystic tumour to the right of the pregnant uterus.

Diagnosis. Pregnancy, together with an ovarian cyst with twisted pedicle.

Operation, October 13th, 1900. Tumour greenish-brown in colour, tubal in origin, with pedicle twisted twice on itself, in the inverse direction of the hands of a watch. Some recent adhesions. Ovary enlarged, not participating in torsion. Recovery. Pregnancy not interrupted.

\section{Pinard. Comptes Rendus de la Soc. d'Obstét. de Gynécol. et de Padiat. de Paris, 1902.}

Torsion of a Hydrosalpinx during pregnancy. Married woman, aged 36, one previous pregnancy. Patient came under Pinard's care June, 1902; last period September 5th to 13th, 1901. History of short attacks of pain in the right inguinal region for five years, worse since the pregnancy started. Admitted for severe pain, nausea, and frequent micturition; later vomiting, diarrhoea, and meteorism; slight icterus. It was decided to induce labour, and deliver as rapidly as possible. Delivery effected in 70 minutes. Temporary improvement, but abdominal section 
undertaken, as arranged before delivery. In the right iliac fossa tumour the size of an orange, a right hydrosalpinx, twisted twice on its pedicle in the inverse direction of the hands of a watch. Ovary normal. Removal of right appendages. Recovery.

6. Kleinhans. Veit's Handbuch, p. 713. Illustrated.

No clinical details of case. Specially interesting from the presence of a hæmatocele due to a left tubal abortion, associated with a hæmatosalpinx on the right side, due to torsion and adhesions. Right tube showed a quite thin isthmic portion, and was then bent down and backwards, with one axial twist. Tube swollen beyond the twist. Contents, dark fluid blood. Wall infiltrated with blood.

7. Harpoth. Centralbl. für Gynäkol., 1900, p. 1,399.

Left tube twisted $2 \frac{1}{2}$ times, "from left to right." Right tube untwisted. Sero-purulent contents of both, but contents sterile. Absence of adhesions which rendered twist possible.

8. Waldo. American Journ. of Obstet., August, 1901.

Single woman, 17 years old. Abdominal pain, etc. No irregularity or change in menstruation.

Diagnosis. ? Appendicitis. At operation, right hæmatosalpinx. Contents entirely fluid, dark in colour, no clots. Ovary drawn up by the tumour, but not involved in twist. Ligature on pedicle slipped, but there was no hæmorrhage; complete strangulation. Twist of pedicle described thus: "Several distinct and complete twists on its long axis." Direction not stated. Left tube and ovary normal. Recovery.

9. Waldo. American Journ. of Obstet., August, 1901.

Married woman, aged 26, no pregnancies. Abdominal pain and tenderness. Menstruation regular.

Dragnosis. Inflamed and adherent ovarian cyst. At operation proved to be a left hæmatosalpinx with "several complete twists" of pedicle. Direction not stated. Ovary not involved and left in situ. Also right tube and ovary. Recovery.

10. Baldwin. American Journ. of Surg. and Gynecol. St. Louis, 1900. I obtained this reference, but have not been able to see the paper.

11. McCann. Lancet, May 9th, 1903. Illustrated.

Married woman, aged 37, sterile, menstruation regular. Four attacks of acute abdominal pain, culminating in very severe attack a fortnight before operation. A swelling on the right side of the uterus, bluish-black in colour. Intestines adherent to it. When pedicle exposed three complete turns observed. Ovary not involved and left in situ. Appendages on opposite side normal. Dilated tube contained blood-clot and dark fluid blood. Tubal wall infiltrated with blood. Sections made from the wall showed chorionic villi, also infiltrated with blood. Recovery.

12. Lewers. Trans. Obstet. Soc. of London. Vol. xliv., p. 362.

A single woman, aged 37. First attack of abdominal pain and romiting December, 1901. A similar attack May, 1902, then continuous pain till September, 1902, when she had a third acute attack. Menstruation 
normal. Physical signs suggesting the presence of two ovarian tumours. This consequently the diagnosis, "and that probably, judging from the history, one had a twisted pedicle." Operation October 9th, 1902. Condition found to be that of double pyosalpinx. The right pyosalpinx was firmly adherent to small intestine and omentum, and slightly adherent to the bladder. When freed from adhesions the dilated part of the tube was found to be attached to the broad ligament by a pedicle twisted several times, which consisted of the undilated inner part of the Fallopian tube. Direction of the twist not stated. The right ovary was not involved, and was not removed. The pedicle of the left pyosalpinx was not twisted. Bacteriological examination failed to demonstrate the tubercle bacillus, but Dr. Lewers states that "though no positive evidence of tubercle was found, he thought that most probably the salpingitis was tubercular." Sepsis and gonorrhoea were fairly excluded by the fact that the patient was a virgin. Uninterrupted recovery.

To proceed to the details of the case under my own observation :-

P.A.C., aged 45, was admitted to St. Thomas's Hospital, February 9th, 1903, under the care of Dr. Cullingworth. She had been a fairly healthy women, but subject to severe and recurring attacks of bronchitis. There was a family history of tuberculosis, her father, sister, and two brothers having died of consumption. Catamenia began at 13, and till two years ago the periods had always been regular, of the 28 day type, and lasting for 3 or 4 days. The patient was married at 19 , and eighteen months later bore a healthy child, the labour and puerperium being normal. Since then she had never been pregnant.

In 1899 she was seized one evening with severe pain in the lower part of the abdomen. The pain was such as to cause faintness and vomiting, but it lasted only a few hours, and she did not see a doctor. So far as the patient can remember no headache or fever, diarrhœa or constipation, accompanied the attack of pain. For two years she was free, and then, in 1901, the attacks returned, but though sharp while they lasted they were of short duration, and never led to her seeking advice until a very severe attack in February, 1903, which immediately preceded her entrance to the hospital. All through there had been no trouble with the bowels, nor with micturition. For the last two years the periods had lost their regularity, recurring at intervals varying from one to three months, but there was absolutely no evidence of any relation between the attacks of pain and uterine hæmorrhage. She had herself noticed no increase in the size of the abdomen, and was quite unconscious of any lump or swelling.

During the last six months the abdominal crises had recurred 
with increasing frequency, at intervals of one month to six weeks, and greater severity. The last attack was the severest of all.

On examination of the abdomen, which was full and rather tense, a swelling could be felt extending from the pubes upwards to a point one inch below the umbilicus. The fingers could be inserted over the borders of the swelling, between it and the anterior superior spines on both sides. There was marked tenderness on the left side. Fluctuation could be obtained.

Per Vaginam. The uterus was lying behind the swelling, no part of which projected into the pelvic cavity. It could be felt lying in front and above the anterior vaginal wall, but it caused no bulging. Bimanually the swelling gave the impression of being a not very tense cyst about the size of an ostrich's egg. It lay with its largest diameter across the abdominal cavity, reaching to within lin. of the ant. sup. spine on the right, and almost as far on the left. High up behind the cervix could be felt a hard fixed nodular swelling, which however did not depress the vaginal vault.

Per Rectum. The mass could be distinetly felt projecting into the bowel from the right side, the projection being itself of the size of a hen's egg, but forming part of a larger mass. An impulse was conveyed directly to the mass projecting into the rectum from the abdominal swelling.

The measurement from the pubes to the upper limit of the tumour was $4 \frac{1}{2}$ inches.

Examination of the chest showed that the heart was healthy, but there was impairment of resonance over the base of the right lung, and a pleuritic friction sound in the same area. Rhonchi were heard over both lungs. The temperature was normal, and the pulse of fair strength and normal rate. The urine was acid, sp. gr. 1,030, with a heavy deposit of urates, but no albumen, sugar, or blood.

A week later (February 16th) a further pelvic examination was made by Dr. Cullingworth. The portion of the tumour extending into Douglas's pouch, and into the vagina above the right fornix, was still almost completely fixed. It was partly cystic, but in part consisted of a harder and more irregular mass, with cords on its surface suggestive (to the touch) of the inflamed veins of a varicocele. Per rectum the swelling conld now be felt distinctly in front of the canal, between it and the upper part of the vagina.

The most probable explanation of the existing condition in the light of the patient's history was that the attacks described represented a series of twists of the pedicle of a right-sided ovarian cyst, the harder and more fixed portions in the pelvis representing 
the parts more immediately affected, and therefore altered by œdema and adhesions, probably also by extravasations of blood.

Three days later the abdomen was opened. The omentum was found adberent to the subjacent viscera. It was carefully separated from its adhesions and pushed upwards. The abdominal mass could now be explored. A large cystic swelling was identified, and its pedicle made out, apparently rising from the left side of the uterus. Some adhesions to bowel and anterior surface of the uterus were broken down, and the tumour brought out through the incision. It was dark, almost black, in colour, and obviously connected with the left appendages. To it was attached an apparently normal ovary. The pedicle was twisted, and the next step in the operation was the unwinding of the twist. To do this the cyst was taken in both hands and turned in the direction of the hands of a watch. The turns were carefully counted, and it was found that there had been $1 \frac{3}{4}$ complete twists of the pedicle (Fig. 1). A blunt pedicle needle, double threaded, was pushed through the mid-line of the pedicle close to the uterine end, and the ligatures interlocked and tied. The pedicle was then cut through and the cyst removed. For greater security the stump of the pedicle, which had been secured by forceps, was encircled by a silk ligature and tied.

The right side of the abdominal cavity was now explored, and deep down in the pelvis another tumour was found. It was brought to the surface, and proved to be a hydrosalpinx of the right tube, with the right ovary flattened against its pedicle, and adherent to it. The pedicle was tied, and the tumour removed, together with a portion of the ovary. On examining the pelvis the uterus was found to be freely movable, and to have returned from its retroflexion to a normal position. The abdomen was closed without drainage.

Convalesence was uninterrupted, the temperature never being above $100^{\circ}$. The patient got up on the eighteenth day, and left the hospital just four weeks after the operation. The last note is dated March 16th. "Progress unimpeded. Uterus in good position and mobile."

Examination of parts removed. The cyst removed from the left side could be seen now to be a hæmatosalpinx, converted into such from a previous hydrosalpinx by the twisting of its pedicle. It formed a large tumour, measuring $5 \mathrm{in.}$ by $4 \mathrm{in}$. in its longest diameters. It was impervious both at the uterine and fimbriated ends. The tube as it left the uterus was much thickened, the wall measuring $\frac{1}{4}$ of an inch. Gradually the lumen dilated, until it reached a point about $2 \mathrm{in}$. from the uterine end, then suddenly it 


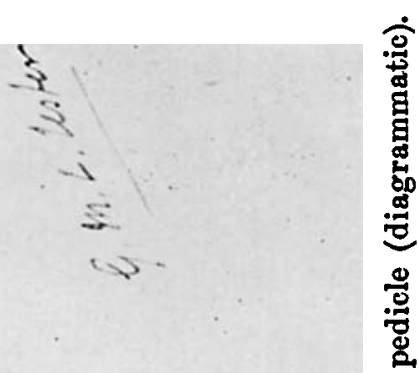

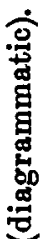

号

$\stackrel{8}{7}$

范

葛

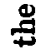

모ำ

胥

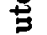

론

8

蒀

$\stackrel{8}{ \pm}$

응

弯

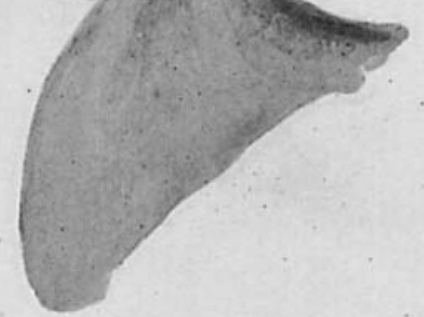

帝

章

$\mapsto$

画 


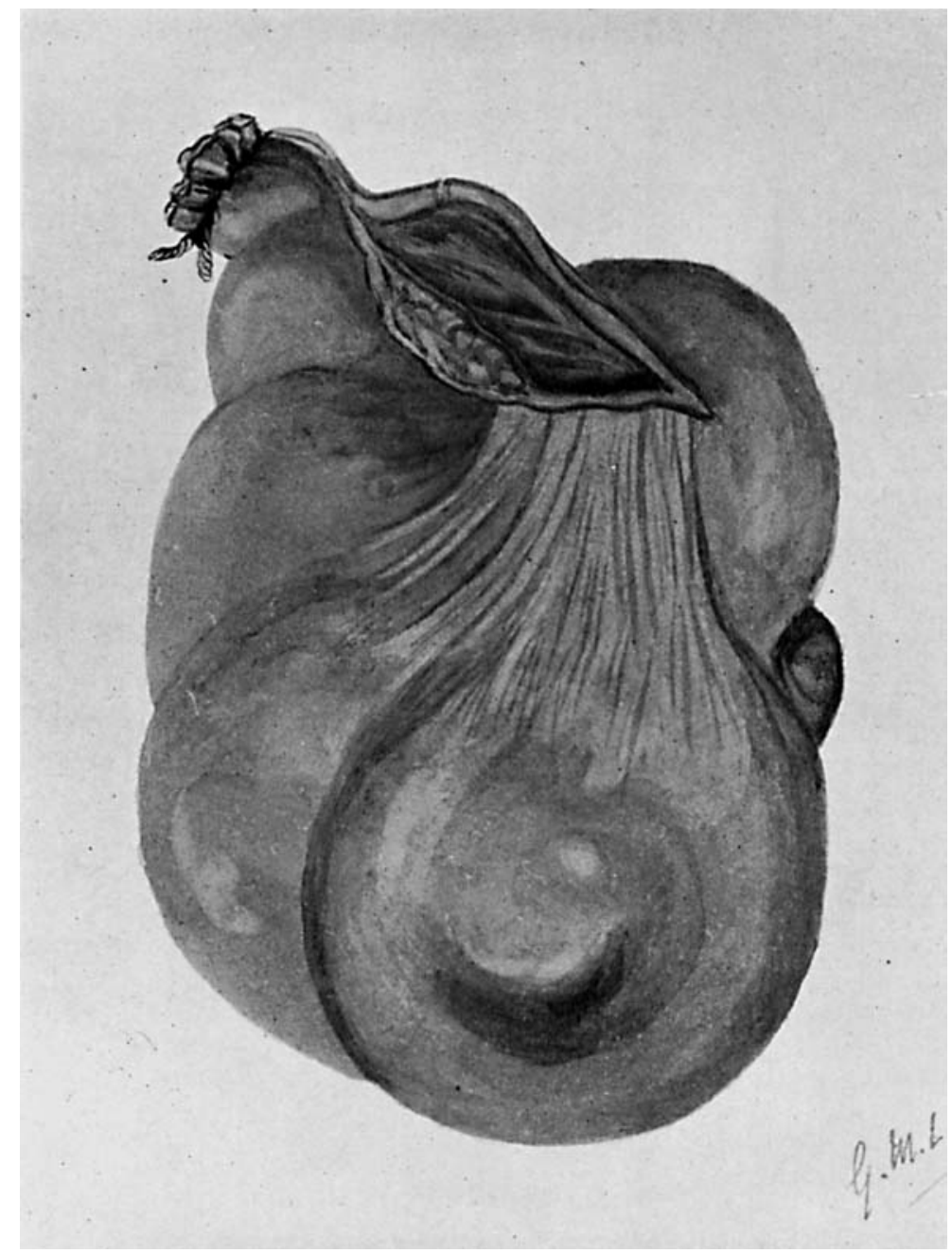

Fia. I1.

External view of the cyst, showing its bossy, irregular character. 


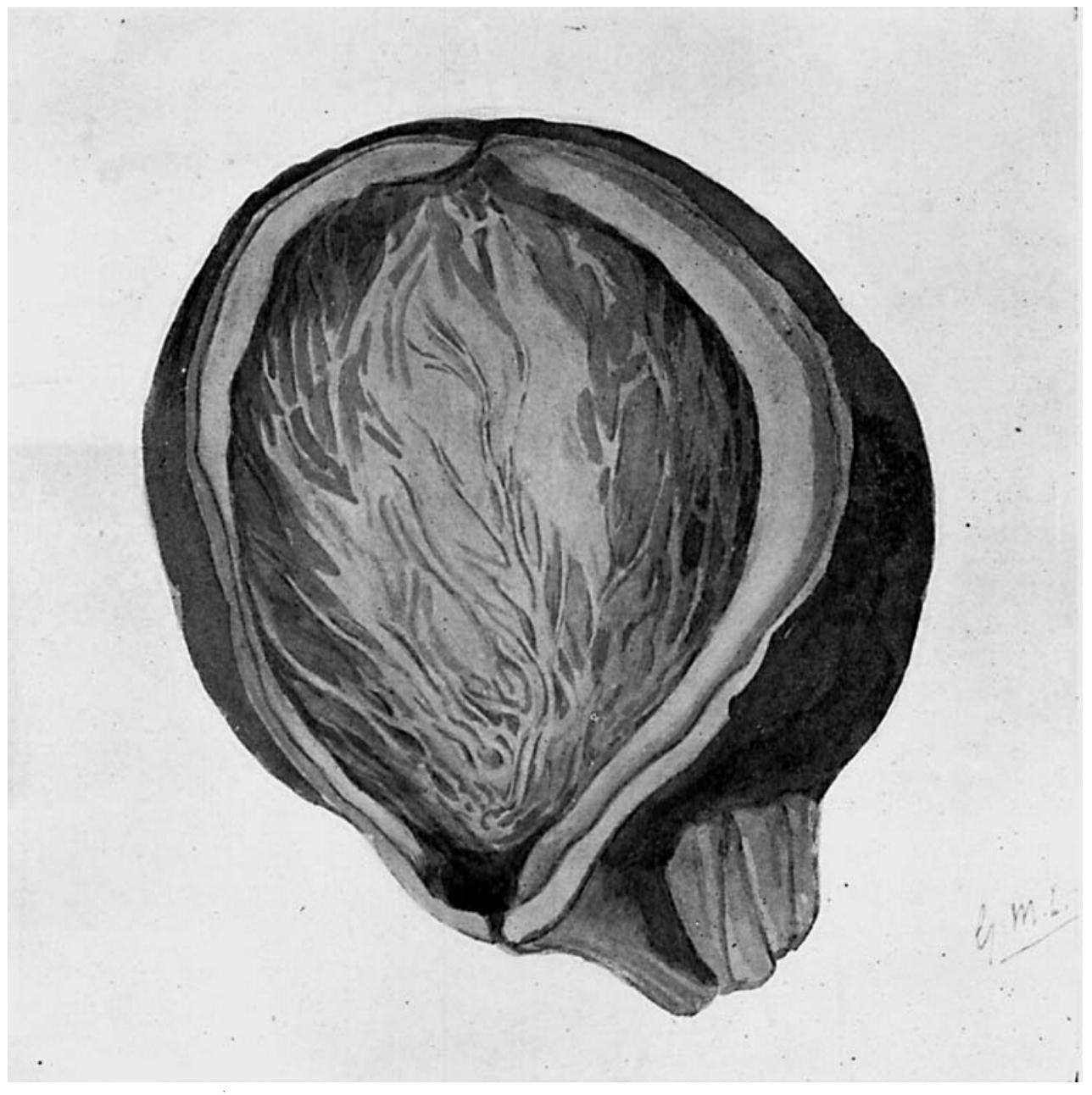

Fig. III.

The interior of the cyst, showing the ribbed appearance, and the wall greatly thickened by hæmorrhage. 
enlarged into a bladder-like cyst, the fimbrix of the tube forming a ribbed network of raised tissue on the internal wall of the cyst (Fig 3). For the greater part of its area the cyst wall was thick and hæmorrhagic, but at the distal end there was an area about the size of a crown which was thin, and free from hæmorrhage.. On section the wall of the cyst was found to consist of a thin outer colourless fibrous layer, a broader middle layer of dark hæmorrhagic material, and an inner thin layer of mucous membrane, which was continuous over the narrow part of the tube, and over the ribs which represent the fimbriæ. This inner layer, with the hæmorrhagic layer, disappeared, to the naked eye at least, on reaching the thin portion of the wall already referred to. The contents of the cyst measured about $140 \mathrm{z}$. The fluid was dark and grumous in character, about the colour of chocolate. It was strongly alkaline, and became solid on boiling. Under the microscope were seen the remains of many red blood cells, and a good deal of amorphous débris resembling broken-down epithelium. No pus cells were seen.

Attached to the external surface of the cyst was the left ovary, slightly hypertrophied and œdematous, but otherwise normal. Near the ovary was a second small cyst, the size of a walnut, filled with dark altered blood. The right Fallopian tube was dilated into a typical hydrosalpinx, measuring in its greatest diameters 3 and 4 inches. The walls of the cyst were thin and transparent. There was evidence of pelvic inflammation in the strong adhesions, which divided the whole cyst into a series of rounded swellings, and matted and fimbriated end to the ovary and to the mesosalpinx. No microscopical examination was made.

The whole problem of torsion in relation to the abdominal viscera is, of course, raised by such a case as this. As is well known, nearly every organ in the abdomen is affected in varying proportions, the liver perhaps being the single exception. Torsions of the bowel, kidney, and testicle are common, and of the spleen not infrequent. Of the female pelvic viscera the ovary is very frequently affected, the tube much more rarely, and the uterus least of all. What is the exciting cause? To this, I think, the answer must be that there is no single exciting cause, but a variety, some intrinsic to the organ affected, and some extrinsic. As regards the first point the relative frequency of torsion in cases of dermoid and solid tumours of the ovary is very suggestive. Storer states that in 248 cases of ovarian torsion collected from various sources there were 43 dermoids and 23 solid tumours. Irregular shape and 
varying weight and consistence must tend to produce disturbance of equilibrium. Freund insists that this is the chief factor in torsions even of high degree. But most observers have regarded the extrinsic causes as of more account. Certain conditions must of course be present to give freedom of movement, of which the most obvious are a certain length of pedicle, and a smooth peritoneum, without adhesions. We shall see a little later how these conditions apply specially to tumours of the Fallopian tubes, but given their presence the actual exciting cause of torsion is still to seek, and the suggestions that have been made are very numerous. In Thornton's cases of torsion of ovarian cysts, pregnancy and labour played a prominent part. Others have suggested the alternate filling and emptying of the bladder and rectum. This last, one would think, might produce a slow progressive twisting (a condition which is by no means rare, and judging from the history was probably present in the case here recorded), but not an acute strangulation. 'The latter must be produced by some more violent agent, such as the sudden descent of the diaphragm, or quick movement of the body as a whole. Quick alterations of abdominal pressure such as are produced by labour or tapping might also be effectual. I think myself that sufficient stress has not been laid on the movements of the diaphragm in producing both kinds of torsion, the acute and the chronic. In the latter case suppose the peritoneum to have lost a little of its lustre. There would then be a certain amount of friction, easily overcome by the active contraction of the diaphragm, but sufficient to resist the much weaker force of its passive relaxation. It is easy thus to imagine a tumour driven slowly onwards, and the twist of its pedicle gradually increased.

It was mentioned above that the presence of a sufficiently long pedicle and the absence of adhesions are the two primary conditions which must be satisfied if torsion is to take place. 'The fact that these conditions can only occasionally be satisfied in tumours of the Fallopian tube explains the rarity of the phenomenon in these cases.

In most cases of salpingitis or pyosalpinx the adhesions are numerous, and torsion consequently impossible. In Cathelin's list of 41 observations there are six of pyosalpinx, but he gives reasons for thinking that these are not primary cases of purulent salpingitis, but rather primary hydrosalpinx, with subsequent hæmato- and pyosalpinx. He points out that in one of the observations of Pozzi the contents are described as a mixture of pus and blood. Of course a pyosalpinx is seen occasionally floating free in the pelvis, and in this case torsion would be as easy as in a hydrosalpinx, but the 
condition is rare. Probably if examined it would prove to be tubercular in origin. Nearly all the cases of tubal torsion recorded are torsions of a hydrosalpinx, converted into a hæmatosalpinx by the torsion, just as hæmorrhage occurs in an ovarian cyst when its pedicle is twisted. The case recorded here is a good example of this, bilateral hydrosalpinx, with conversion of the left hydro- into a hæmatosalpinx by twisting of the pedicle. Besides hydrosalpinx several cases of torsion of tubal gestations have now been recorded, e.g., by A. Martin, Pozzi, and McCann, one or two also of malignant disease (Stroganoff and Warnek), and Hartmann and Reymond published a curious case of twisting of a tube apparently quite healthy save for the hæmorrhagic infiltration produced by the twist itself.

Besides the absence of adhesions it is necessary for the tumour to have a distinct pedicle, and it is much more easy for it to rotate when the pedicle is long and thin, and the mobility free. These conditions are satisfied when the swelling is situated in the ampulla of the tube, and not in the isthmus, and when sufficient growth of the tumour has taken place for it to have obtained an abdominal rather than a pelvic situation. The former condition is almost a sine qua non, the latter not absolutely necessary, but certainly favourable. Where the exact localisation of the tumour in relation to the parts of the Fallopian tube is mentioned in the recorded cases it is almost without exception a dilatation of the ampulla, connected with the cornu of the uterus by a long, and usually thin, pedicle.

Two Varieties of T'orsion. Legueu particularly has insisted on the slow or chronic variety of torsion. It is by no means so common as the acute form. It cannot truly be said that there is any hard and fast line between the two forms, but clinically the cases are very different, urgent symptoms in the one leading to immediate operation, and in the other a succession of twists with only short, if sharp, attacks of pain, occurring at intervals perhaps for years. In my own case the first attack of abdominal pain occurred four years before her admission to hospital, and during the last two years the attacks had gradually increased in frequency and severity. It seems probable that these attacks were produced by successive twists, leading ultimately, but very slowly, to complete strangulation of the pedicle, and acute symptoms.

Direction of Rotation. The methods of recording this are so various and confusing that it is difficult to arrive at any exact conclusion as to the usual direction of the twist. "From left to right," or "from behind forwards" are phrases loosely employed. I 
think undoubtedly the best method of describing the direction of torsion is that usually employed by the French writers, namely, " in the direction, or in the inverse direction of the hands of a watch." It must be understood that the face of the watch is towards the tumour, the back towards the uterus, but no other conditions need be observed. In my case the twist was unravelled by turning the tumour in the direction of the hands of a watch. The original twist was therefore in the inverse direction. As it was a left hydrosalpinx this follows Kustner's "law of torsion." But there are so many exceptions to this law that it can hardly be considered to have much validity, as regards torsions of hydrosalpinx at any rate. Cathelin gives the following table in this connection :-

R. Side

L. Side

In the direction of the hands of a watch ........ 2 cases. In the inverse direction ......................... 5 ,

In the direction of the hands of a watch ........ 2 ,"

In the inverse direction ........................ 3 ,

These figures afford no support whatever to Kustner's so-called law. They point, on the contrary, to there being no definite rule as regards the direction of the twist.

Results of Torsion. The number of turns varies greatly in the recorded cases, from a half twist (i.e., through $180^{\circ}$ ) up to $4 \frac{1}{2}$ twists. The actual number of twists is really of little importance. What is important is the tightness of the twist, and consequent strangulation. It is possible to have a complete twist without any interference with the blood supply, but the usual thing is for the veins to be compressed, and as a natural result of the impeded venous return there is a rapid increase in the size of the tumour, with hæmorrhage both into the cyst and between the layers of its wall. Occasionally the strangulation is so complete that necrosis or gangrene occurs. In one or two recorded cases the twist has resulted in the complete, or nearly complete, separation of the pedicle. Occasionally bleeding takes place into the peritoneal cavity, and a hæmatocele is formed, as in Praeger's first case.

The usual tumour of the tube to undergo torsion is a hydrosalpinx, and it is transformed by the hæmorrhage induced by the torsion into a hæmatosalpinx. The contents are usually fluid, clots being only rarely found. The colour is variously described as dark red, blackish, resembling chocolate, or like the contents of a hæmatocele. Pus is occasionally found, but we have mentioned 
already that it may be doubted if these are primary torsions of a pyosalpinx. It seems more reasonable to suppose that suppuration has occurred after the twist, as often happens in the case of ovarian cysts.

The ovary is involved in the twist in roughly one-third of the cases. I have found it in 11 out of 30 in which the point is specifically mentioned. Rarely other organs are affected. In one case of Hartmann's the uterus was twisted a half-turn on itself. In another observation the bladder was nipped between a fibroid uterus and the twisted hydrosalpinx. Adhesions are of course common, to the broad ligament, to the pelvic peritoneum, to bowel, and to omentum. Hartmann and Reymond in France, and Praeger in Germany have made careful microscopical examinations of the wall of the cyst, and of the pedicle at the seat of torsion. As might be expected the sections show litle more than hæmorrhages in all directions. The arteries in the pedicle remain permeable, at least many of them, though the walls are dissociated by interstitial hæmorrhages, and the vasa vasorum very dilated. The veins on the other hand are dilated and thrombosed. The microscopical appearances vary of course with the degree of torsion. As Cathelin puts it there are four stages, congestion, hæmorrhage, mortification, and separation.

Influence of Age and Pregnancy. As might perhaps be expected, the age at which this form of torsion occurs is that of full genital activity. Out of 42 cases in which the age is noted 34 were between 20 and 40 (13 of these between 20 and 30 , and 21 between 30 and 40). There were 7 cases between 40 and 50, and 1 case under 20 , an unmarried girl of 17. No case over 50 has yet been recorded. Pregnancy does not seem to play a very important part as a predisposing agent. Out of 38 cases 10 were nulliparous, and 15 had had only one confinement. At the same time several cases are directly associated either with pregnancy or the puerperium. In Cathelin's list of 41 cases there is only one operated on actually during pregnancy, but in the few cases that I have added two coincided with a four months' gestation, and were operated upon without any interruption of the pregnancy, and one was dealt with immediately after artificial delivery at practically full term. Multiple pregnancies weaken the abdominal wall, and would naturally favour torsion if a tumour of the tube were present; but there is the other side to the picture, namely, that diseases of the tubes are commonly associated with sterility, absolute or relative. The figures given above afford another illustration of this fact. 
I propose now to consider shortly the more clinical aspect of these cases, under the headings of symptoms, diagnosis, and treatment.

Symptoms. The most prominent symptom, common to all the cases, is abdominal pain, of a severe type, situated in the lower part of the abdomen, and in nearly all cases referred to the side on which the twist has occurred. The pain is sudden in onset, and very sharp, often leading to faintness and even syncope. In many cases, of which the one recorded here is a good example, there is a history of many previous attacks of pain, usually of short duration, and not so severe as the final attack which leads to operation. These previous attacks may be associated with slight twists of the pedicle, not leading to complete strangulation, or on the other hand they may be, when not severe, due to slight attacks of salpingitis and pelvic peritonitis, and so connected rather with the primary disease which leads to the formation of the salpingitic tumour than with the torsion of the pedicle. In certain cases the tumour itself has been recognised for months before the severe and final attack of pain. This occurred in Praeger's two cases for example. In my own observation though the woman had not herself noticed any enlargement of the abdomen, nor was she conscious of any lump, it is certain that the double hydrosalpinx must have been present for some time, and would have been found had she submitted herself to examination. In those cases where a tumour has been noted previously there are distinct changes observed in it occurring with the acute attack of pain, changes exactly similar to those occurring in an ovarian cyst when its pedicle becomes twisted, i.e., enlargement, tenderness, and loss of mobility.

The pain, though usually situated in the lower abdomen, radiates sometimes to the inguinal region and legs, to the hypochondrium, or to the loins. This has led to wrong diagnoses, such as appendicitis.

There is usually little or no interference with menstruation. In Cathelin's series out of 17 cases where the point is mentioned in only four was there any irregularity. Occasionally however there are irregular hæmorrhages between the periods. It is doubtful whether these are due to the original disease of the tube, or to the torsion. Certainly in the analagous cases of torsion of the ovary some irregular bleeding is a fairly frequent symptom.

In my own case the periods had lost their regularity for two years, recurring at intervals varying from one to three months, but there was no evidence of any relation between the attacks of pain 
and uterine hæmorrhage. It must too be noted that the patient was 45 years of age.

Peritonitic symptoms are well marked. Vomiting is commonly noted, of varying severity, but never fæcal. The belly is usually distended, and constipation is the rule, sometimes absolute even to the absence of flatus, leading in two cases to the diagnosis of intestinal obstruction. On the other hand diarrhoa is noted in a few cases. The bladder is occasionally interfered with, leading to frequency of micturition. Retention of urine occurred in one case.

Slight fever is sometimes present, but it is not the rule. The pulse rate is generally quickened, and the face has a somewhat anxious look.

As regards the physical signs, the abdomen is usually distended, and tender to the touch. On gentle palpation a tumour can often be made out, fluctuating, and more or less fixed, according to the amount of local peritonitis excited by the torsion. In the cases where a tumour has been observed beforehand, augmentation of volume occurs.

The presence of a tumour in the abdomen is very characteristic of this lesion, in contrast to the usual pelvic situation of disease of the Fallopian tubes. This is doubtless due to the fact that torsion is much more likely to occur when the hydrosalpinx has reached such a size as to become an abdominal tumour than when it is confined within the narrow limits of the pelvis.

Vaginal examination does not commonly afford much information, save in the few cases where the tumour is situated in the pouch of Douglas. In my own observation the tumour did not enter the pelvis, but could be felt lying in front of and above the anterior vaginal wall. This situation has been noted in three other cases.

Bimanually it is possible, if the patient is not too tender, to separate the tumour from the uterus in the great majority of cases, and localise it to the appendages. A careful examination should always be made to determine if possible the condition of the opposite appendages, as disease in that region might suggest the tubal rather than ovarian origin. In one case, published by Warnek, he was able to feel the twisted pedicle both by the abdomen and per vaginam, but this must be very rarely possible. The tumour was on the right side. He noted disease of the left appendages also, but still did not come to a correct diagnosis, regarding it as a case of right ovarian cyst with twisted pedicle, and left salpingitis.

Diagnosis. So far as I have been able to discover an absolutely correct diagnosis has never yet been made. The symptoms and signs 
detailed above show an almost exact correspondence with the symptoms and signs of a twisted ovarian tumour, and this is the most common diagnosis. Sometimes the characteristic signs are not present, and examination is very difficult from the tenderness of the patient, or the presence of another abdominal tumour, such as pregnancy or a fibroid uterus. In these cases the operation is of an exploratory nature, and there is a provisional diagnosis only, usually of salpingitis or appendicitis.

I think the fact that a diagnosis has never been made or even suggested is due to the possibility of the condition not being present to the mind of the examiner. The cases are no doubt rare. Pozzi, in reporting his four cases in 1900 , stated that he had never met with the occurrence before 1899 , and the case reported here is the first to come under Dr. Cullingworth's observation. Still the number of recorded cases has now reached above fifty, and all since BlandSutton's premier observation in 1891. Given the following conditions and signs I think the diagnosis should be at least suggesteda fluctuating abdominal tumour, not rising above the umbilicus, associated with paroxysmal attacks of pain, culminating in a very severe attack, with vomiting and constipation, and if the tumour has been observed before, increase of size, tenderness, and some loss of mobility.

The great difficulty is to distinguish this from torsion of an ovarian cyst. It cannot be done with certainty. But the shape of the tumour may be suggestive. An ovarian cyst is usually rounded, the twisted hydrosalpinx much more irregular. (In my own observation the tumour only reached to one inch below the umbilicus, and lay with its largest diameter across the pelvis.) If the abdomen were too tender for palpation percussion might be of aid. Instead of the definite upper convex limit of dulness there might be an irregular curve, corresponding rather to the retortshaped hydrosalpinx than to the circular or oval ovarian cyst. Of course it is only in the case of small ovarian cysts that the question of differential diagnosis could arise. In tumours above the umbilicus no one would suggest disease of the tubes. Bimanual examination might lead to the discovery of bilateral disease, with acute symptoms on one side. This would be a further point in favour of tubal rather than ovarian torsion.

If the case is seen for the first time during the acute attack the severe abdominal pain, collapse, vomiting, \&c., suggest either colic (hepatic, renal, or intestinal) or appendicitis. If a tumour is present a mistake is not likely to be made, but where this is absent, or small 
and not discovered from the extreme abdominal tenderness, the difficulty is great. Localisation of the seat of pain is sometimes of value, and the absence of particular symptoms such as tenderness at McBurney's point or jaundice, would help in the differential diagnosis. Ruptured extra-uterine gestation might be suggested, but if watched for a little it is seen that there are not the symptoms of great internal hæmorrhage, nor on the other hand the physical signs of the formation of a hæmatocele. As we have said before it is only rarely that a twisted hydrosalpinx forms a tumour situated in the pelvis.

From one point of view perhaps a correct diagnosis is a matter of no very great importance. The surgeon may be satisfied that operative interference is called for, and is ready to open the abdomen and prepared to deal with any condition he may find present. But the value of a careful attempt to make a diagnosis beforehand is $I$ think undoubted, and I trust that before long a case may be recorded in which not only will the patient's life have been saved by a timely operation, but also that the condition will have been diagnosed correctly before the abdomen was opened.

Treatment. This requires little discussion. It is necessarily surgical, and I think no one would dispute that the abdominal is the correct route. The incision should be made in the mid-line, or a little to one side, preferably the side of torsion. After removing the twisted tube the appendages of the other side should be carefully examined. Where the tube is obviously diseased it will of course be removed. One ovary should if possible be left in situ. It is a more difficult matter to decide what to do when the other tube is not obviously diseased. Cathelin in his discussion of treatment decides in favour of " une castration bilatérale," leaving only a fragment of ovary, with a secondary hysteropexy, but he allows that if the other appendages are absolutely healthy it may be better to leave them so as to give the patient a chance of a subsequent pregnancy. I think this should certainly be the rule, and that after removal of the twisted tube the other should be dealt with conservatively, by separation of adhesions for example, wherever possible.

With regard to "washing out" and drainage, each case must be considered separately. Though the practice of surgeons differs, I think it may be said that nowadays drainage should be avoided, unless there is some very positive indication for its use.

The prognosis after operation is favourable. So far only three deaths have occurred in just over fifty cases. 
Articles:--

Bibliography.

Hartmann and Rermond. Annales de Gynécol., 1894 and 1898.

Maillard. "De la torsion des Salpingites," Thése de Paris, 1897-98.

PRARGEle. Archiv für Gynäkol., 1899. Bd. Iviii.

Liggueu. La Presse Médicale, Paris, January 20, 1900.

STorer. Boston Med. and Surg. Journal, 1896. Vol. ii., p. 461.

Cathelin. "De la torsion des Hydrosalpinx. Rev. de Chirur., 1901, pp. 253 and 406 . At the end of this article is a table of 41 observations with Bibliography.

Cases recorded in paper:--

1 Praeger. Archiv für Gynälol., 1899. Bd. Iviii.

2 Ibid.

3 Pozzr. Comptes Rendus de la Soc. d'Obstét. de Gynécol. et de Padiat. de Paris, April, 1900.

4 Pinard and Paqux. Comptes Rendus de la Soc. d'Obstét. de Gynécol. et de Padiat. de Paris, October, 1901.

5 Pinard. Comptes Rendus de la Soc. d'Obstét. de Gynécol. et de Pcediat. de Paris, December, 1902.

6 Kleinhans. Veit's Handbuch, p. 713.

7 НАпротн. Centralbl. für Gynäkol., 1900, p. 1,399.

8 WaıDo. American Jonirn. of Obstet., August, 1901.

9 lbid.

10 Baldwin. American Journ. of Surg. and Gynecol., 1900. St. Louis

11 McCann. Lancet, May 9th, 1903.

12 Lewers. Trans. Obstet. Soc. of London. Vol. xliv., p. 362. 